\title{
NEW DATA ON SOME MOTH SPECIES (LEPIDOPTERA) FROM MT. JADOVNIK AND MILEŠEVKA RIVER CANYON (WEST SER- BIA)
}

\author{
PREDRAG JAKŠIĆ ${ }^{1 \star}$, ANDREW KING ${ }^{2}$ \\ ${ }^{1}$ Faculty of Natural Sciences and Mathematics, University of Priština, Kosovska Mitrovica, Serbia \\ ${ }^{2}$ Grovewood Close 18, Chorleywood, Hertfordshire, WD3 5PU, United Kingdom
}

\begin{abstract}
Data on six species of Lepidoptera significant for the fauna of Serbia, as the data on one quite rare species in Serbian fauna are presented.
\end{abstract}

Keywords: Lepidoptera, West Serbia.

\section{INTRODUCTION}

The Lepidopteran fauna of in Serbia is insufficiently known. This is especially true of the fauna of the microlepidoptera. In terms of the number of identified species, Serbia lags significantly behind neighboring countries. As far as we are aware, there is no organized and systematic research being undertaken by academic or similar institutions. Fragmentary data come mostly from foreign authors. Some domestic authors act together in NGOs, or independently. Their results are relatively modest, but still significant. Recently organized studying Lepidoptera in the region has been created as "Balkans Moths Project" by several scientists. Some of the results contain numerous new data on Lepidoptera of Serbia published in Plant et al. (2017) and Beshkov et al. (2020). As the part of this project, monograph on butterflies of Mt. Jadovnik will be published soon (Jakšić (2020), in print). The aim of this paper is to add new data on several species rare in western Serbia.

\section{MATERIAL AND METHODS}

Moths were sampled at light during night using a white sheet illuminated by $125 \mathrm{~W} \mathrm{Hg-Li} \mathrm{mercury} \mathrm{bulb.} \mathrm{The} \mathrm{species} \mathrm{are} \mathrm{listed}$ according to their relevant families, according to Zhi-Qiang Zhang (2011).

Fieldwork in protected areas was undertaken in agreement with permits provided by the Ministry of Environment, Mining and Spatial Planning, Republic of Serbia, No. 353-01-1559 dated on 8.6.2011; 353-01-1070, dated on 12.6.2012; 353-01637/2013-08 dated on 10.6.2013 and 353-01-916/2014-08 dated on 29.5.2014.; 353-01-356/2015-17 dated on 27.04.2015.; 353-01389/2016-17 dated on 08.4. 2016. and 353-01-763/2019. 04 dated on 23.05.2019.

Photos of in situ specimen were taken by the second author, using a "Nikon D-3200" camera. All the material (specimens and genitalia slides) is deposited in the collection of P. Jakšić in Belgrade (CPJB).

\section{RESULTS}

Six species belonging to five families are presented in this paper.

Fam. Hepialidae Stephens, 1829

Korscheltellus lupulina (Linnaeus, 1758)

New data: Sopotnica village, 986 m, 2 males, 1 June 2014, Genitalia checked, slides SR-2641 and SR-2652; Kašanj village, 1276 m, 1 female, 8 June 2016.

Literature data for Serbia: Abafi-Aigner (1910), Beshkov (2017), Kereši \& Almaši (2017), Tot et al. (2018).

A moth of grassland. The subterranean larvae feed on the roots of grasses and herbaceous plants.

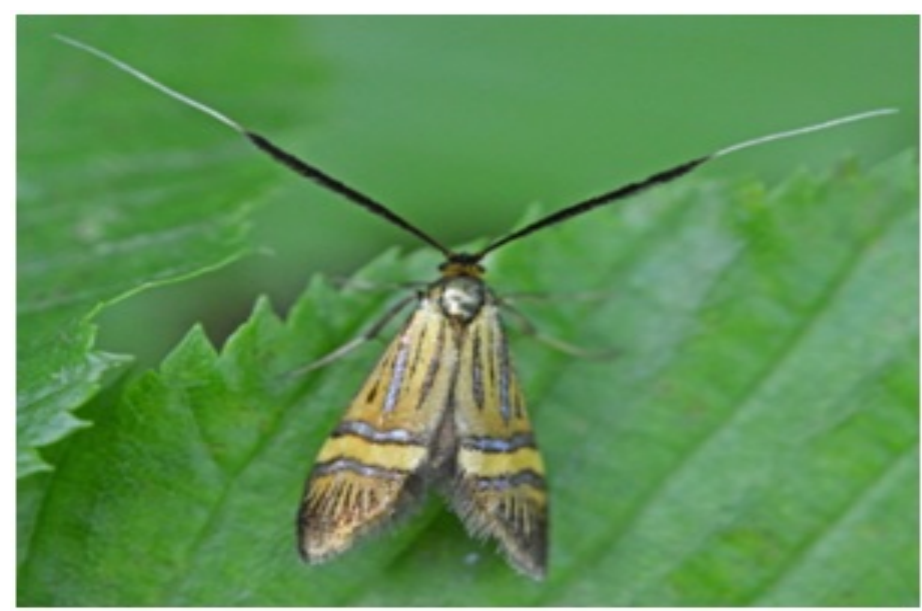

Figure 1. Nemophora degeerella complex Sopotnica village, 986 m, 26 June 2016.

Fam. Adelidae Bruand, 1851

Nemophora degeerella complex 
New data: Sopotnica village, 986 m, 26 June 2016, (Fig. 1).

Literature data for Serbia: Rebel (1911), Rebel (1914), Rebel (1917), Rebel \& Zerny (1931), Rotschild (1912-1917), Szent-Ivany (1945), Zečević (2002).

A moth of open woodland and scrub. The males form leks 'dancing' aerially beside bushes in the sunshine. The larvae initially feed on various flowers and then build portable cases and move around on the ground, feeding on dead vegetation.

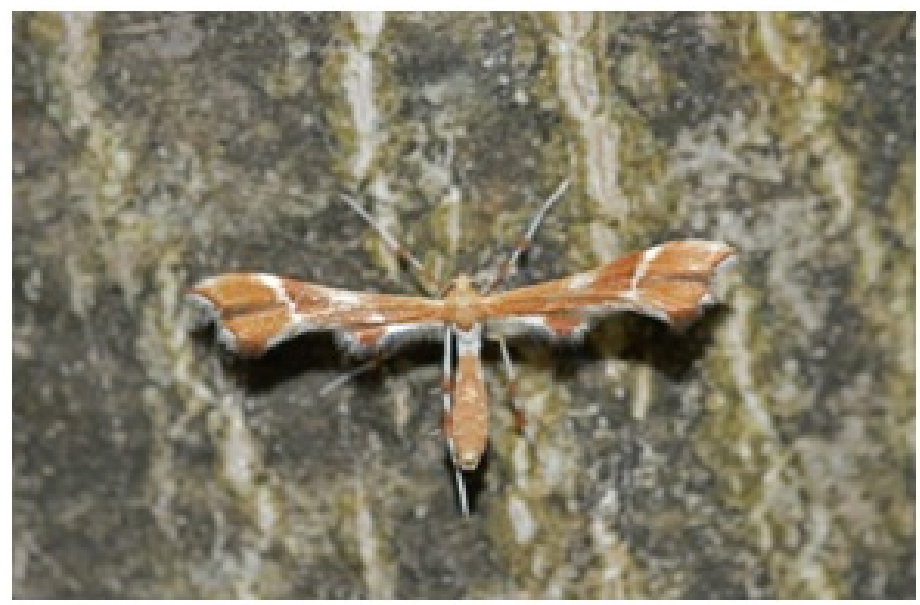

Figure 2. Cnaemidophorus rhododactyla (Denis Schiffermüller, 1775) Mileševka Canyon, 585 m, 14 June 2016.

Fam. Pterophoridae Latreille, 1802

Cnaemidophorus rhododactyla (Denis \& Schiffermüller, 1775)

New data: Mileševka Canyon, 585 m, 14 June 2016 (Fig. 2).

Literature data for Serbia: Arenberger \& Jakšić (1991), Gielis (2003), Guelmino (1996), Rebel (1914), Rebel \& Zerny (1931), Rotschild (1912-1917), Stojanović (2012), Stojanović et al. (2014), Tot et al. (2018), Zečević (1999), Zečević (2002),

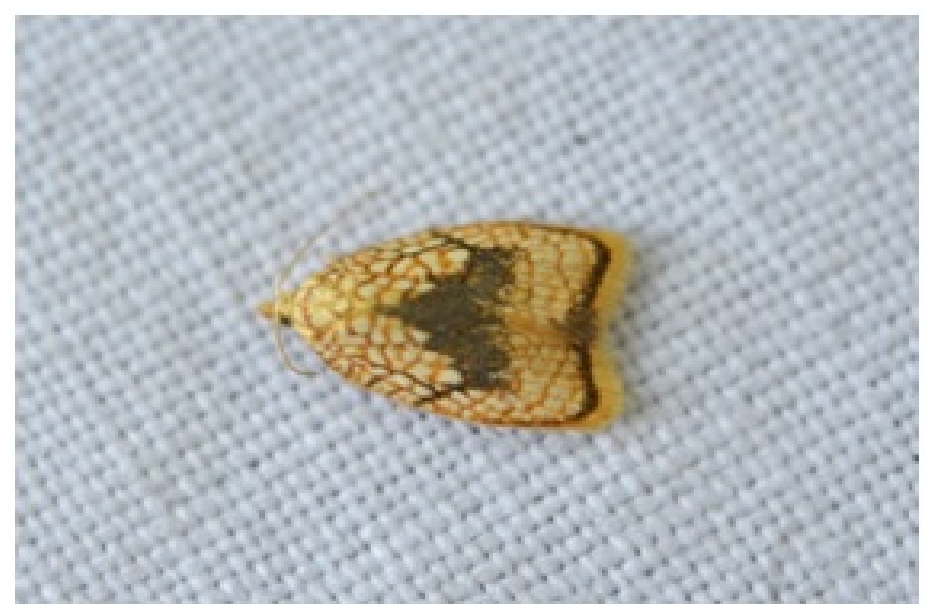

Figure 3. Acleris forsskaleana (Linnaeus, 1758) Mileševka Canyon, 585 m, 26 June 2016.

A moth of woodland margins and scrub, as well as on open limestone areas where the host plant grows. Larval food-plant the flower-buds and shoots of Rosa canina. Matović (1986) and Obratov (1992) reported presence of this species.

Fam. Tortricidae Latreille, 1802

Acleris forsskaleana (Linnaeus, 1758)

New data: Mileševka Canyon, 585 m, 26 June 2016 (Fig. 3).

Literature data for Serbia: Đurić \& Hric (2015), Stojanović (2012), Stojanović et al. (2014), Tot et al. (2018), Zečević (1999), Zečević (2002).

A moth of woodland, hedgerows and scrub. Nocturnal. Larvae spin together flowers and later leaves of the food-plants, which are Acer campestra, A. pseudoplatanus, A. platanoides and possibly other Acer spp. Matović (1986) and Obratov (1992) reported presence of these species.

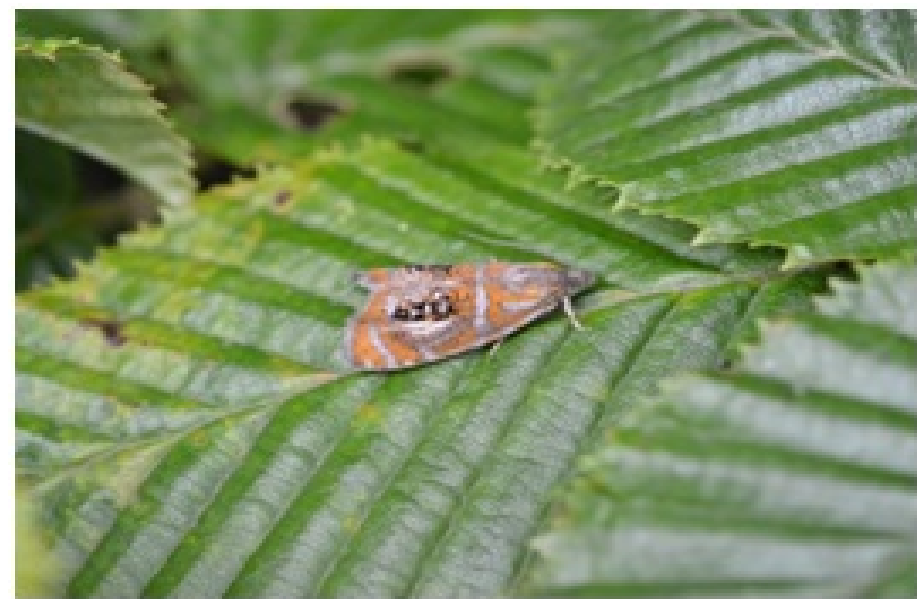

Figure 4. Olethreutes arcuella (Clerck, 1759) Šćepanica, 1139 m, 15 June 2016.

Olethreutes arcuella (Clerck, 1759)

New data: Šćepanica, 1139 m, 15 June 2016, (Fig. 4).

Literature data for Serbia: Rebel (1914), Rebel (1917), Rotschild (1912-1917), Stojanović (2012), Stojanović et al. (2014), Tot et al. (2018), Zečević (1999), Zečević \& Vajgand (2001), Zečević (2002), Živojinović (1950).

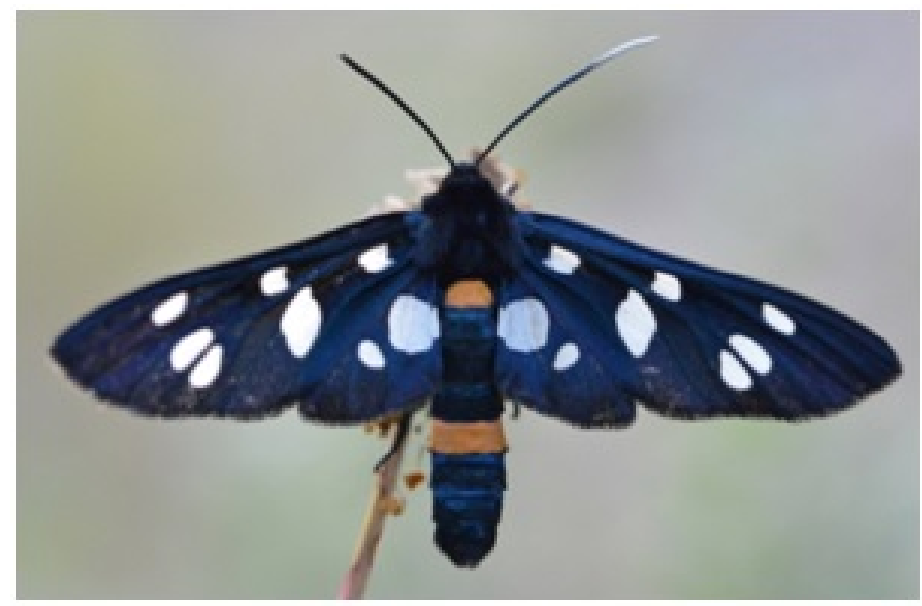

Figure 5. Amata kruegeri marjana (Stauder, 1913) Mileševka Canyon, 585 m, 26 June 2016. 
A moth of open woodland and woodland margins. Flies mostly in sunshine and also at night. Larvae feed, on the ground, on withered and decaying leaves.

Fam. Erebidae Leach, [1815]

Amata kruegeri marjana (Stauder, 1913) (Nomenclature according to De Freina (2008))

New data: Mileševka Canyon, 585 m, 26 June 2016 (Fig. 5).

Literature data for Serbia: Obraztsov (1966), Vojvodić (2011).

New species for Mt. Jadovnik. Day-flying over grassland. The larvae feed on Plantago, Rumex, Galium and Taraxacum spp. and probably other low-growing plants.

\section{REFERENCES}

Abafi-Aigner, L. 1910, Adaléka Magyar Tengermellék. Horvátország és Dalmáczia lepkefaunájához. Rovartani lapok, 17(34), pp. 55-57, (5-8), pp. 71-105, Budapest.

Arenberger, E. \& Jakšić, P. 1991, Pterophoridae (Insecta, Lepidoptera). In: Nonveiller, G. (ed.): Fauna Durmitora 4, pp. 225242. Titograd.

Beshkov, S. 2017, Contribution to knowledge of the Lepidoptera fauna of the Balkan Peninsula. Entomologist's Record and Journal of Variation, 129(1), pp. 9-33, 9 plates, 54 figs.

Beshkov, S., Plant, C., Nahirnić, A., King, A., \& Jakšić, P. 2020, A contribution to knowledge of Balkan Lepidoptera: moths collected in May-June 2018 in Austria, Slovenia, Serbia, North Macedonia and Albania. Entomologist's Record and Journal of Variation, 132, pp. 24-45.

De Freina, J. 2008, Über die Biologie, Morphologie, Phänologie und Taxonomie von Amata (Syntomis) kruegeri (Ragusa, 1904) (Lepidoptera: Arctiidae, Syntominae, Syntomini). Nachr. entomol. Ver. Apollo, N.F., 28(3/4), pp. 97-107.

Gielis, C. 2003, Pterophoroidea Alucitoidea (Lepidoptera). In: World Catalogue of Insects, Apollo Books, 4, pp. 1-198.

Guelmino, J. 1996, Zenta környékének állatvilága. II. Gerinctelen állatok (Životinjski svet Sente). Zenta. Dudás Gyula Múzeumés Levéltárbarátok Köre 1-79+11 tabs.

Jakšić, P. 2020, Dnevni leptiri Jadovnika, Mileševke i susednih područja (Lepidoptera: Papilionoidea). Prirodnjački muzej u Beogradu, Posebna izdanja, 47, pp. 110.

Kereši, T. \& Almaši, R. 2017, Nocturnal Lepidoptera in the vicinity of Novi Sad (Northern Serbia). Acta entomologica serbica, 14(2), pp. 147-162.

Matović, M. 1986, Vegetacija kanjona Mileševke. Monograph. Glas Polimlja, pp. 1-63.

Obratov, D. 1992, Flora i vegetacija planine Zlatar. Dissertation. Faculty of Biology. Belgrade.

Obraztsov, N. 1966, Die Palaearktische Amata-Arten (Lepidoptera, Ctenuchidae). Verö_entlichungen der zoologischen Staatssammlung Munchen, 10, pp. 1-383, 30 tabs, 79 figs.

Plant, C., Jakšić, P., Beshkov, S., \& Nahirnić, A. 2017, A contribution to knowledge of the Balkan Lepidoptera. Some Pyraloidea (Lepidoptera: Crambidae Pyralidae) encountered recently in southern Serbia, Montenegro, the Republic of Macedonia and Albania. The University Thought - Publication in Natural Sciences, 7(2), pp. 1-27. https://doi.org/10.5937/univtho7-15336

Rebel, H. 1911, Die Lepidopterenfauna von Herkulesbad und Orsova. Eine zoogeographische Studie. Annalen des K.K. Naturhistorischen Hofmuseums, 25(3/4), pp. 253-430, figs. 116, tab. 1(figs. 1-17).

Rebel, H. 1914, Adatok Magyarország lepkefaunájához, (Beitrage zur Lepidopterenfauna Ungarns). Rovartani Lapok, XXI(1-3), pp. 27-53.

Rebel, H. 1917, Lepidopteren aus Neumontenegro. Sitzungsberichte der Akademie der Wissenschaften mat.-nat. Klasse, 126, pp. 765-813.

Rebel, H. \& Zerny, H. 1931, Die Lepidopterenfauna Albaniens. Denkschriften der Akademie der wissenschaften inWien. Math. Nat. Klasse, 103, pp. 38-159+Taf. I., Wien.

Rotschild, C. 1912-1917, Adatok Magyarorszag lepkefaunajahoz (Beitrag zur Lepidopterenfauna Ungarns). Rovartani Lapok, XVI, pp. 130-148; XVIII, pp. 36-43; XIX, pp. 21-29, pp. 167180; XX, pp. 66-91, pp. 170-175; XXI, pp. 27-47, pp. 72-77.

Stojanović, D. 2012, Taksonomsko-faunistička studija leptira (Insecta: Lepidoptera) Fruške gore. Disertacija. Biološki fakultet, pp. 1- 621. Beograd.

Stojanović, D., Ćurčić, S., \& Tomić, M. 2014, Fauna Lepidoptera Nacionalnog Parka „Tara“ Deo prvi - Microlepidoptera. Novi Sad, Bajina Bašta i Sremska Kamenica.

Szent-Ivany, J. 1945, Faunistische und ökologische Angaben über die Adelinen (Lepidopt.) des Karpatenbeckens mit Beschreibung zwei neuer Arten. Fragmenta Faunistica Hungarica, VIII(1-4), pp. 7-10.

Tot, I., Matić, B., Husarik, J., \& Jovanov, A. 2018, Leptiri Spomenika priroder "Slapovi Sopotnice" i okoline zaštićenog područja (Insecta: Lepidoptera). Zbornik radova Spomenika prirode "Slapovi Sopotnice", pp. 17-33.

Vojvodić, L. 2011, Collection of Butterflies by Stanko Radovanović at the National Museum in Kikinda (Serbia). Bulletin of the Natural History Museum, 4, pp. 131-156, 6 figs.

Zečević, M. 1999, Fauna leptira (Lepidoptera, Microlepidoptera) Timočke Krajine. Razvitak, XXXIX(201-202), pp. 54-58.

Zečević, M. 2002, Fauna leptira Timočke Krajine (Istočna Srbija). Bakar Bor i Narodni muzej Zaječar, pp. 1-307.

Zečević, M. \& Vajgand, D. 2001, Podaci iz kartoteke prof. dr Mihaila Gradojevića o fauni leptira (Lepidoptera) Srbije i Makedonije. Sveske Matice srpske, 37, pp. 34-78.

Zhi-Qiang Zhang, E. 2011, Animal biodiversity: An outline of higher-level classification and survey of taxonomic richness. Zootaxa, 3148, pp. 1-237.

Đurić, M. \& Hric, B. 2015, Unapređeni uvid u noćne leptire Ovčarsko-Kablarske klisure. Beležnik Ovčarsko-kablarske klisure, 6(1), pp. 59-67, 2 figs.

Živojinović, S. 1950, Fauna insekata šumske domene Majdanpek. SAN, Institut za ekologiju i biogeografiju, 2, pp. 1-262. 